\title{
Network model and analysis of the spread of Covid-19 with social distancing
}

\author{
Parul Maheshwari ${ }^{1 *}$ and Réka Albert ${ }^{1,2}$
}

\author{
${ }^{*}$ Correspondence: \\ ppm5104@psu.edu; \\ parul.dkm@gmail.com \\ ${ }^{1}$ Department of Physics, The \\ Pennsylvania State University, \\ University Park, PA 16802, \\ USA \\ Full list of author information \\ is available at the end of the \\ article
}

\begin{abstract}
The first mitigation response to the Covid-19 pandemic was to limit person-to-person interaction as much as possible. This was implemented by the temporary closing of many workplaces and people were required to follow social distancing. Networks are a great way to represent interactions among people and the temporary severing of these interactions. Here, we present a network model of human-human interactions that could be mediators of disease spread. The nodes of this network are individuals and different types of edges denote family cliques, workplace interactions, interactions arising from essential needs, and social interactions. Each individual can be in one of four states: susceptible, infected, immune, and dead. The network and the disease parameters are informed by the existing literature on Covid-19. Using this model, we simulate the spread of an infectious disease in the presence of various mitigation scenarios. For example, lockdown is implemented by deleting edges that denote nonessential interactions. We validate the simulation results with the real data by matching the basic and effective reproduction numbers during different phases of the spread. We also simulate different possibilities of the slow lifting of the lockdown by varying the transmission rate as facilities are slowly opened but people follow prevention measures like wearing masks etc. We make predictions on the probability and intensity of a second wave of infection in each of these scenarios.
\end{abstract}

Keywords: Epidemic spreading, SIR model, Social network, Network science, COVID19, Reproduction number

\section{Introduction}

The spread of the novel SARS-CoV-2 virus has posed a new challenge to the scientific community and society at large on mitigating the spread of a viral infection. The lack of vaccines or therapies for the virus calls for non-pharmaceutical intervention in the course of disease spread. As a result, the immediate response from most of the world was to limit the contact among people in the population by requiring them to stay at home. The person-level effect of these measures is to maintain physical distance with everyone except co-habiting family members and reduce social interactions. The expected effect of this distancing is the reduction in the spread of the virus, widely referred to as "flattening the curve". While the real-time data shows a reduction in the rate of spread in situations where social distancing was strictly followed (Hopkins 2020), analysis to predict the exact effect of the mitigation measures will be useful to understand the possibility of

(c) The Author(s) 2020. Open Access This article is licensed under a Creative Commons Attribution 4.0 International License, which permits use, sharing, adaptation, distribution and reproduction in any medium or format, as long as you give appropriate credit to the original author(s) and the source, provide a link to the Creative Commons licence, and indicate if changes were made. The images or other third party material in this article are included in the article's Creative Commons licence, unless indicated otherwise in a credit line to the material. If material is not included in the article's Creative Commons licence and your intended use is not permitted by statutory regulation or exceeds the permitted use, you will need to obtain permission directly from the copyright holder. To view a copy of this licence, visit http:// creativecommons.org/licenses/by/4.0/. 
a second wave of infection and choose an optimal strategy to minimize the effect of the pandemic while minimizing the economic impact of the restrictions. Different epidemiological modeling approaches modeled this reduced contact as modified parameters of the rate of spread of the infection. Most of these epidemiological models are compartmental models that assume a fraction of the population as either susceptible (S), infected $(\mathrm{I})$, or recovered/dead $(\mathrm{R})$ and model the rate of change of these continuous variables (S, I, and R) assuming full mixing among individuals (Gaubert et al. 2020; Chen et al. 2020; Toda 2020). There are also network-based epidemiological models that represent human-human interactions as edges and model the spread of the disease from individual to individual by infecting a fraction of the network neighbors of an infected node (Block 2020; Chinazzi 2020; Kraemer 2020; Gutin, et al. 2020; Aleta, et al. 2020). The fraction infected is varied as the population starts to follow social distancing measures.

Here, we present a network SIR model where the individual-to-individual spread of the disease is simulated compared to the traditional SIR models that study the S, I, R fractions of the whole population. Each individual-to-individual interaction is represented as an edge between two nodes (individuals) in an interaction network. In this model, the effect of lockdown or social distancing leads to a modification in the network structure. We construct a network for a given population size so that it comprises of two different kinds of edges, one that is deleted in lockdown and other that continues to exist during lockdown. These two kinds of edges denote the two different kinds of interactions we have in our daily lives. The edges that are preserved during lockdown denote interactions with family members and essential service providers and the edges that are deleted during lockdown denote other "non-essential" interactions like workplace interactions, socializing at events and club meetings, etc. We find that this way to simulate the social distancing in the population gives promising results in terms of reduction of the spread of the virus. We then explore different periods of lockdown with different strategies of phasing out of the lockdown and find interesting insight on the possibility of a second wave as a result.

\section{Methods}

\section{Construction of the network}

We construct networks of 10,000 nodes. Each node corresponds to a person and an edge between nodes corresponds to a non-zero probability of disease transmission between the two persons. Each person is assumed to be a part of a family. The network nodes do not include anyone under the age of 18 on the assumption that the disease has little effect on children. Family sizes range from 1 (with frequency of 30\%), 2 (with frequency of $35 \%), 3$ (18\%) to 4 (17\% frequency); this data was obtained and approximated from (O'neill and Chen 2020). Each family is a clique in the network. We assume that $70 \%$ of the population is made up by working individuals. We approximate this number from official sources ensuring that they are a good pre-pandemic estimate for early 2020 (EPR $2020 \mathrm{a}, \mathrm{b})$. We assume that $20 \%$ of the working population are essential workers. We construct a scale-free network (Barabási and Albert 1999) with a minimum degree of 2 and degree distribution $\mathrm{P}(\mathrm{k}) \sim \mathrm{k}^{-3}$ among the non-essential working population. We assume that the network among the essential working population is random (Erdös 1960) with average degree 25. Additionally, there are edges that represent interaction between the 
population and essential workers. This network covers the interactions that happen due to essential activities like grocery shopping, healthcare, etc. This is a random network with the probability of an edge to an essential worker set to 0.2 . Finally, some edges are added to the network to cover social interaction outside of families and workplaces in the population. This is a random network with average degree of $\sim 1.3$ (Table 1 ).

\section{Dynamics of the spread}

Each 10,000-node network is initialized with 5 infected nodes, selected randomly, and the rest of the nodes are susceptible. Each infected node can infect its neighbors (i.e. nodes that are connected to the infected node by an edge) with a certain transmission probability. The transmission probability depends on whether the infected person is symptomatic or asymptomatic. Since symptomatic people are likely to visit the doctor, and then they isolate themselves at a hospital or at home, we assume that their transmission probability to essential workers (i.e. health workers) is 0.05 and their transmission probability to the general population is 0.005 . We assume the transmission probability for asymptomatic infected persons is 0.05 . Additionally, we assume that $80 \%$ of the infected nodes are asymptomatic while the other $20 \%$ are symptomatic. From the data on Covid-19 death rates, we assume a $5 \%$ mortality among the infected nodes. These $5 \%$ form a subset of the symptomatic patients. After the other $95 \%$ recover, $85 \%$ become immune to the disease while the remaining $10 \%$ become susceptible again. The number of days it takes for a person to recover from the infection varies from 1 to 35 days, following a normal distribution with a mean of 17 days and standard deviation of 4.8 days. Similarly, the number of days after which a patient loses their life to the disease varies from 5 to 24 days according to a normal distribution centered at 14 days and standard deviation of 3.2 days. In the unmitigated situation, an infected node can spread the infection to any of its neighbors. During a lockdown (i.e. period when the population is following social distancing and stay-at-home order), only certain edges of the network are retained for the spread of the disease. These edges correspond to family interactions, interactions among essential workers, and interactions between the population and essential workers. We run this simulation for many graphs, and for each graph, we run the spread process multiple times. In total, we take an average over 50 runs and report the results (Table 2).

Table 1 Table of parameters used for the construction of the network

\begin{tabular}{ll}
\hline Parameter & Value \\
\hline$n$ (network size-number of nodes) & 10,000 \\
Employment-to-population ratio & $70 \%$ \\
Employees in the essential services & $20 \%$ \\
$\lambda$ (exponent of the scale-free degree distribution) & 3 \\
Average degree of essential services network & 25 \\
Population-to-essential worker edge probability & 0.2 \\
Average degree of additional social interactions & 1.3 \\
\hline
\end{tabular}

Most of these parameters are an estimation from various sources to reflect the US population pre-pandemic. The network size of 10,000, while clearly more representative of a small town than of a country, allows computationally efficient simulation and rich outcomes of the disease spread. The parameters can be changed in the code (see "Methods" section) 
Table 2 Table of parameters specific to the disease and the nature of its spread

\begin{tabular}{ll}
\hline Parameter & Value \\
\hline Initial number of infections & 5 \\
Symptomatic-to-essential transmission & 0.05 \\
Symptomatic-to-population transmission & 0.005 \\
Asymptomatic-to-all transmission & 0.05 \\
Ratio of symptomatic cases & $20 \%$ \\
Probability of mortality & $5 \%$ \\
Probability of immunity & $85 \%$ \\
Mean of recovery period & 17 days \\
Mean time to death & 14 days
\end{tabular}

These parameters reflect an intuitive estimation at the time of model construction with the intention to study general trends. The values of these parameters can be changed in the code (see "Methods" section)

\section{Reproduction number of the disease}

The reproduction number is a measure of the expected number of cases directly generated by one infected case. The basic reproduction number is this measure in a population where everyone is susceptible, and the effective reproduction number $\left(\mathrm{R}_{\mathrm{t}}\right)$ is for a population where some fraction of the population is immune. In this network modeling, we calculate the reproduction number by counting the number of people an infected person spreads during the time they were sick. For example, consider node A who gets infected on day 1 and node infects 2 persons on day 2, 1 person on day 3, 2 persons on day 4. Node A recovers on day 5 and cannot infect any more persons. So, node A infected 5 persons in total and hence the reproduction number for node $\mathrm{A}$ is 5 . To calculate the disease's effective reproduction number for day 1 , we find the reproduction number for each node that got infected on day 1 and take the average. Hence, the reproduction number of a day is the average reproduction number for every person that caught the infection on that day.

The code for the network generation, spread of the disease, and calculation of the reproduction number of the disease, is freely available on GitHub: https://github.com/ parulm/spread_lockdown.

\section{Results}

\section{Network model}

The constructed network is a combination of scale-free and random networks with multiple cliques of sizes 1-4. Hence, in the resulting network most of the nodes have a low degree $(<10)$ but there also are some hubs (with degree $>100$ ). As illustration we show a 100-node network constructed by this method (Fig. 1). This sample network has average degree of $\sim 5$ with maximum degree of 21 . It has 10 connected components, the largest of which contains 86 nodes.

The centrality measures of a network generated using this method vary as a result of the randomness and stochasticity in the generation process, however the network still has some common features. The degree distribution of the network shows two peaks, 


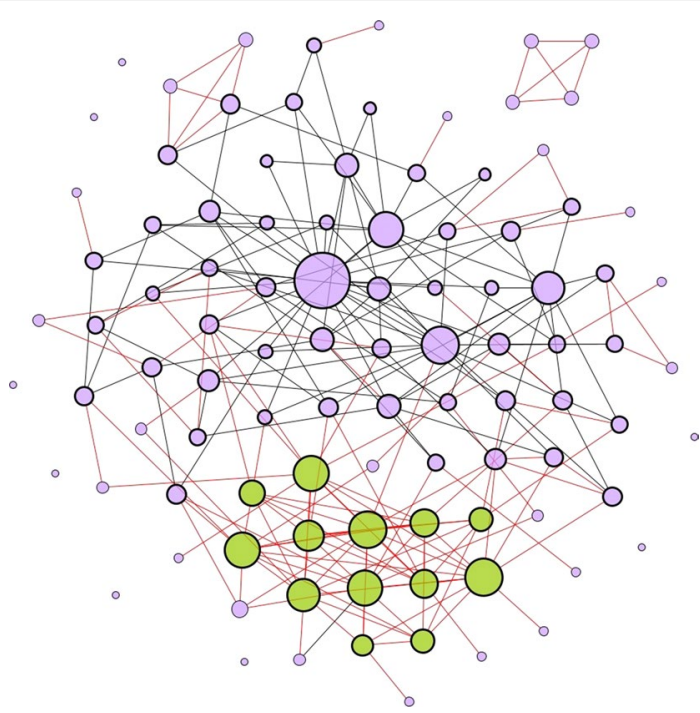

Fig. 1 Illustration of the result of the network generation method on a population of 100 individuals. The edges represent interactions within families, workplaces, and social interaction. The red color edges are the ones that are preserved in the lockdown while the black color edges are deleted. The 4-cliques with red edges, as well as a fraction of 3-cliques with red edges represent families. Node size denotes the degree of the node with isolated nodes having the smallest size; this illustrates that the network contains high-degree hubs. The nodes that have a thick border denote the people who are working. Nodes in green color denote essential workers. The cluster of green nodes shows a high interaction among essential workers

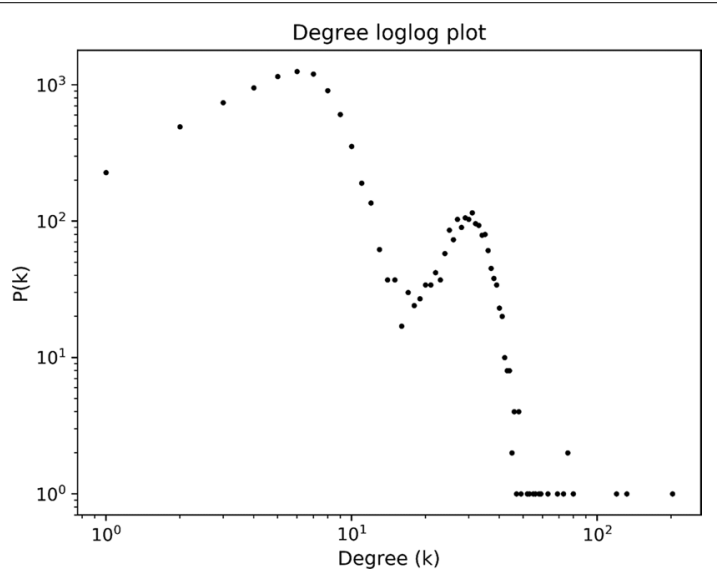

Fig. 2 The degree distribution of a network generated with 10,000 nodes shows two peaks representing the combination of workplace interactions. The first peak at $k \sim 6$ is due to the scale-free network of non-essential workplace interactions and the second peak at $k \sim 25$ is coming from the random network of essential workplace interaction. The high-degree tail represents the hubs, i.e., the individuals who have many interactions, who could therefore act as super-spreaders if infected

one at low degrees (6-7) and the other centered around 25, the average degree of the essential workplace network; it also has a significantly long tail, see Fig. 2. The average 
degree of a 10,000 -node network is $\sim 10$ and the maximum degree is 184 . The network has nearly 50 connected components, the largest of which contains 9950 nodes.

\section{Simulations of spread}

In the absence of mitigation measures, the disease either dies off (in $73 \%$ of the simulations) or spreads in the population. In the simulations where the epidemic takes off, the effective reproduction number starts at a high value (larger than 4), as expected from the theory of epidemic spreading (see Fig. 3). In this case, the disease completes its course in $\sim 80$ days, infecting almost everyone. The peak of infection is around day 30 , when $\sim 45 \%$ of the population is infected at the same time. This disease time course leads to $\sim 400$ deaths, with very few deaths after day 80 . The reproduction number peaks at $\sim 7$ around day 3 and goes to below 1 around day 20 . Nearly $60 \%$ of the population becomes immune to the disease but over $30 \%$ of the population remains susceptible. Since herd immunity needs that at least $70 \%-80 \%$ of the population be immune (D'Souza and Dowdy 2020), the remaining susceptible population could be affected by a second wave of the disease sparked from outside of the network. In conclusion, letting the disease run its course unmitigated is not expected to benefit society in any way. We note that the number of deaths is only valid if the assumption of a constant death rate (of 5\%) holds. It is likely that after day 10 , when the hospitals start getting overwhelmed, the patients do not get all the medical attention they need, thus the death rate may increase.

\section{Lockdown is fixed to 3 months}

If a lockdown is implemented for 3 months, which is longer than the natural duration of the unmitigated outbreak, we find that it almost completely avoids the possibility of a second outbreak. We evaluate 90 day lockdown periods with different starting times: day 5 (when $\sim 1 \%$ of the population is infected), day 10 (when $\sim 6 \%$ of the population is infected), and day 15 (when $\sim 20 \%$ of the population is infected). All these cases lead to

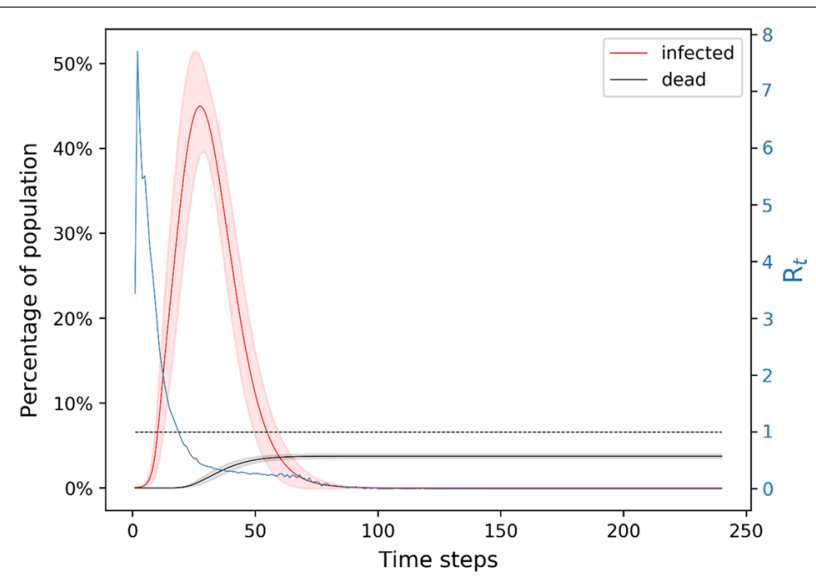

Fig. 3 Unmitigated spread of the disease in the network model. The plot shows for each time step the number of nodes in the network that are infected in red color and the number of nodes that are dead in black color. The reproduction number is shown in blue with the scale shown on the right $y$-axis. The $x$-axis gives the timesteps. The shaded area around the line plots is marked by the standard deviation obtained by running the simulation $\sim 1000$ times. The disease completes its course in $\sim 80$ days, infecting about $70 \%$ of the population 

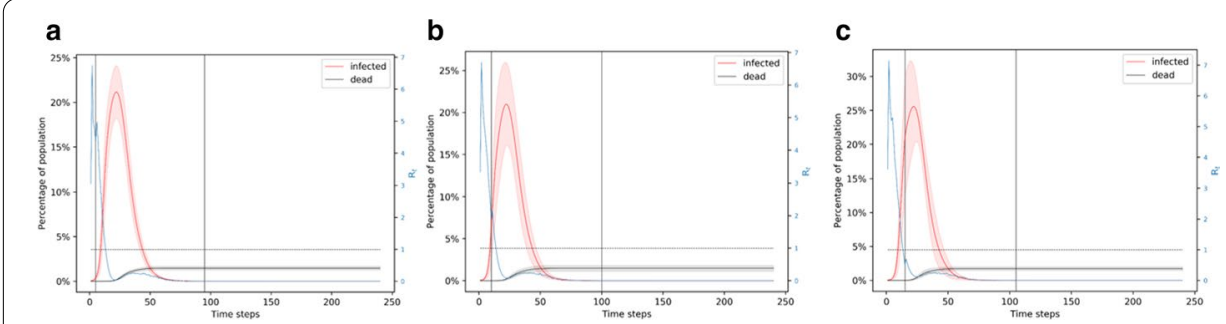

Fig. 4 Simulation of the 90-day lockdown cases. a Lockdown is from day 5 to day 95 . b Lockdown is from day 10 to day 100. c Lockdown is from day 15 to day 105. A 90-day lockdown prevents a second wave of infection. Comparing panels $\mathrm{A}$ and $\mathrm{C}$, we note that a later lockdown leads to a higher peak and more deaths. In all the cases, the peak in the number of infections is foreshadowed by a peak in the reproduction number

more than 150 deaths, with the number of deaths being higher if the lockdown starts later. Shown in Fig. 4a, when the lockdown is from day 5 to day 95, the infection peaks at $20 \%$ of the population. In nearly $1 \%$ of the simulations, there is a second wave of infection which peaks at less than $5 \%$ of the population infected around the same time. This wave of infection peaks nearly 50 days after the end of the lockdown. After 240 days, this scenario leads to a total of 150 deaths which is $1.5 \%$ of the population. Shown in Fig. $4 \mathrm{~b}$, when the lockdown is from day 10 to day 100 , the infection peaks to a little over $20 \%$ of the population. This scenario leads to nearly $0.7 \%$ of the simulations showing a small second wave of infection that affect a very small fraction of the population. After 240 days, this scenario leads to a total of 155 deaths. Figure 4c shows the scenario when lockdown is from day 15 to day 105 . Nearly $100 \%$ of the simulations in this case lead to only the first wave of infection which peaks to $\sim 25 \%$ of the population. After 240 days, this leads to $\sim 175$ deaths.

A high peak of infection implies that the medical facilities will be overwhelmed, which is likely to cause a surge in the number of deaths. While these simulations show that a 90-day lockdown is beneficial for mitigating a widespread effect of the pandemic, they also show that the earlier a lockdown starts, the less the number of deaths and the less pressure on medical facilities. It is also interesting to note that an earlier lockdown leads to a slightly higher possibility of a second wave of infection since a smaller fraction of the population becomes immune. However, despite two waves of infection, the number of deaths is still less if the lockdown starts earlier. In all of these cases, the reproduction number peaks at $\sim 6$ around day 3-4, denoting the high infection spread in the following few days and it goes to below 1 around day 20. These results show that a 90-day lockdown starting the earliest possible would be the most efficient way to mitigate the effects of the disease through social distancing measures. However, due to the adverse economic effects of a lockdown, it is useful to find equally effective strategies that are shorter than a 90-day period. Hence, we next explore the effect of a 60-day lockdown.

\section{Lockdown is fixed to 2 months}

When the lockdown is fixed to 60 days and the starting time is varied, we find that a second wave of infection becomes more likely than in the 90-day lockdown case. Like the 90-day lockdown case, we explore 60-day lockdown for starting times of day 5 (infection at $1 \%$ ), day 10 (infection at 6\%) and day 15 (infection at 20\%). The simulation of the 

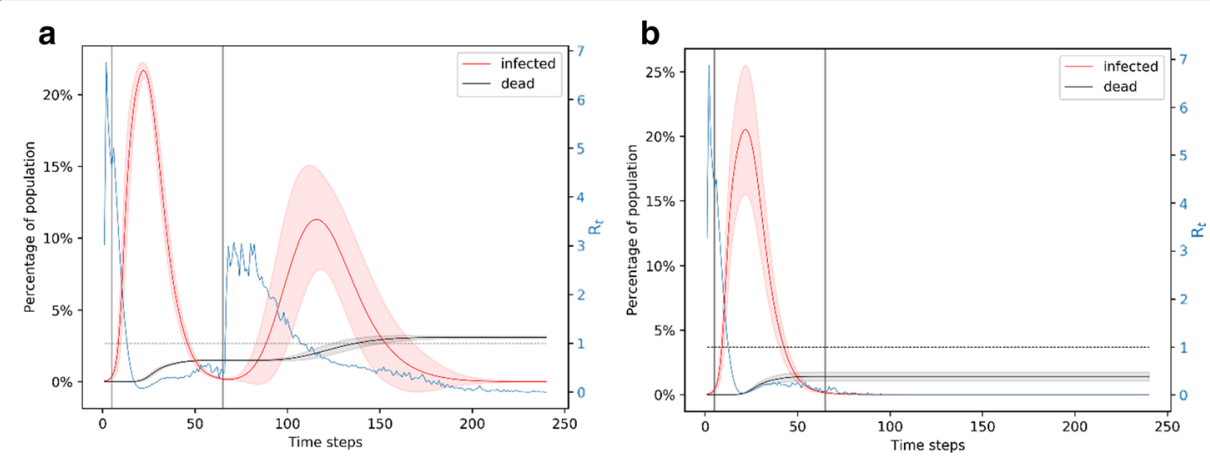

Fig. 5 Simulation of the 60 -day lockdown from day 5 to day 65 . a Representation of the $\sim 40 \%$ of simulation results where there is a second wave of infection when the lockdown is from day 5 to day 65 . The effective reproduction number increases dramatically after the end of the lockdown, foreshadowing the second peak of the infection. $\mathbf{b}$ Representation of the $\sim 24 \%$ simulation results where there is no second wave of infection for the lockdown period of day 5 to day 65 . The effective reproduction number stays below 1 after the lockdown is lifted

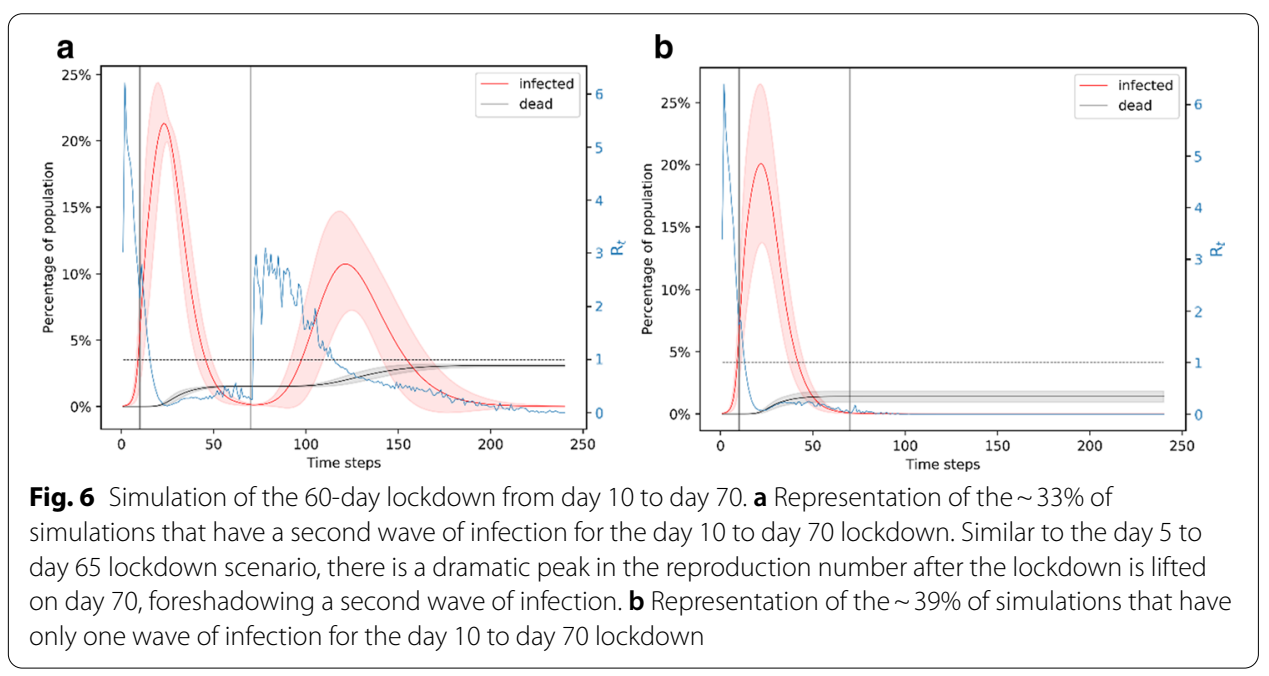

lockdown from day 5 to day 65 shows that there is a second wave of infection in $40 \%$ of the cases (Fig. 5a) and only one wave of infection in $24 \%$ of the cases (Fig. 5b); the remaining $36 \%$ of cases show no spread. The first wave of the infection in response to a day 5 to day 65 lockdown peaks around day 20 and infects nearly $20 \%$ of the population at the same time - this can be seen in both Fig. 5a, b. On day 65, when the lockdown is lifted, around $\sim 1 \%$ of the population is infected. As shown in Fig. $5 \mathrm{a}$, the second wave of infection peaks around day 110 and it is highly varying across simulations. On average, the peak of this wave infects another $10 \%$ of the population at the same time. Simulations with two waves of infection lead to a total of $\sim 300$ deaths which is $3 \%$ of the population. Simulations with only one wave of infection lead to a total of $\sim 150$ deaths. On average, the day 5 to day 65 lockdown scenario leads to $\sim 250$ deaths after 240 days.

We next explore the case when the lockdown is from day 10 to day 70 . This results in $~ 33 \%$ of simulations showing a second wave of infection (Fig. $6 a$ ), $~ 39 \%$ of simulations show only one wave of infection (Fig. $6 \mathrm{~b}$ ) and the remaining $28 \%$ of the simulations 
show no spread. The second wave of infection peaks around day 110 and infects another $10 \%$ of the population at the same time. Two waves of infection lead to $~ 310$ deaths and only one wave of infection leads to $\sim 155$ deaths. On average, the day 10 to day 70 lockdown scenario leads to $\sim 220$ deaths after 240 days. The day 15 to day 75 lockdown scenario leads to two waves of infection in $\sim 16 \%$ of the simulations (Fig. $7 \mathrm{a}$ ), only one wave of infection in $\sim 58 \%$ of the simulations (Fig. $7 \mathrm{~b}$ ) and no spread in the remaining $26 \%$ of the simulations. In this case, the second wave of infection peaks around day 120 and infects another $\sim 7 \%$ of the population at the same time. Two waves of infections lead to $\sim 300$ deaths and only one wave of infection leads to $\sim 180$ deaths. On average, the day 15 to day 75 lockdown scenario leads to 200 deaths.

In all these results, there is at least one peak for the effective reproduction number and this first peak is $\sim 6$ around day $3-4$. In the results that show a second wave of infection, $\mathrm{R}_{\mathrm{t}}$ goes to below 1 during the lockdown but increases again after the end of lockdown; it peaks $\sim 20$ days before the peak in the number of infectious people. From these simulations, we can see that the probability of a second wave of infection is lower if the lockdown starts later. However, the first wave of the infections gets increasingly worse if the lockdown starts later. It makes sense to start the lockdown earlier to minimize the effects of the first wave of infection, but we need other mechanisms to lower the effect of the second wave of infection. This can be done in various ways. For example, impose a second lockdown in response to the second wave of infection and so on until a consequent wave of infection is too small to affect the population adversely. Alternately, instead of lifting the lockdown after a fixed time-period, the lockdown can be lifted when the number of infected cases goes below a fixed threshold. If the number of cases crosses this threshold again, the lockdown is imposed again. In the next section, we consider another similar scenario where after the end of the first lockdown, the population resumes normal life gradually.

\section{Phasing out of the lockdown}

Here, we consider a slow phasing out of the lockdown. That is, facilities reopen slowly after the end of the lockdown and the population strictly adhere to social distancing,
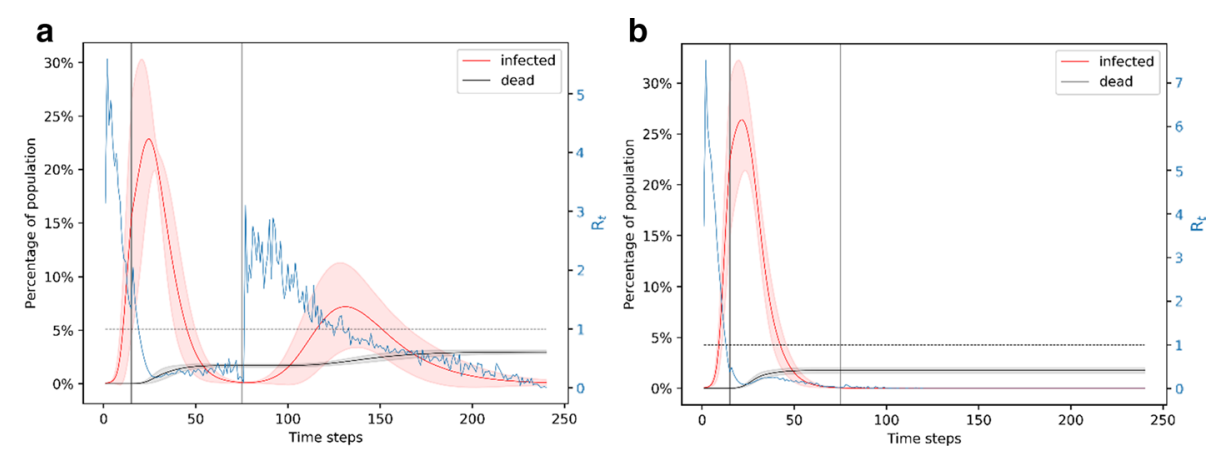

Fig. 7 Simulation of the 60-day lockdown from day 15 to day 75 . a Representation of the $\sim 16 \%$ of simulations that have two waves of infection for the day 15 to day 75 lockdown. The peak in the reproduction number after day 75 foreshadows a second wave of infection. Comparing with Figs. 5 and 6 , we note that the second wave of infection is less intense when the lockdown starts later. $\mathbf{b}$ Representation of the $~ 58 \%$ of simulations that have only one wave of infection for the day 15 to day 75 lockdown 
wearing masks and washing hands, etc. We implement this by restoring the network to its original state, that is, including all edges but reducing the transmission probability after the lockdown ends. The transmission probability then gradually increases to the original transmission probability in the window between the end of the lockdown and the day normalcy is resumed. We assume that the lockdown starts on day 5 and ends on day 65 and we consider three rates of increase in the transmission probability following the end of the lockdown.

In the first case, shown in Fig. 8a, b, the network follows a linearly increasing transmission probability from 0.01 on day 65 to 0.05 on day 80 . This results in $\sim 20 \%$ of the simulations having a second wave of infection, shown in Fig. 8a. This second wave peaks around day 125 and infects nearly $10 \%$ of the population at the same time. The plot in this case (Fig. 8a) is very similar to the plot in Fig. 5a. However, the benefit of the gradual decrease of mitigation is reflected in much lower likelihood of the existence of a second wave (20\%) compared to the likelihood when we resume to normalcy right after the end of the lockdown (40\%). This case leads to an average of $\sim 300$ deaths. Figure $8 \mathrm{~b}$ shows the simulation results when there is only one wave of infection; this case leads to an average of $\sim 150$ deaths. The gradual resumption to normalcy on day 80 results in a lower overall death count at $\sim 200$ compared to the $\sim 250$ with sudden resumption of the original transmission rate (Fig. 5a, b).

In the case shown in Fig. 9a, the lockdown is from day 5 to day 65 and we resume to complete normalcy on day 95 . The network follows a linearly increasing transmission rate from day 65 to day 95; on day 95, the transmission rate reaches the normal $5 \%$. This case results in $\sim 6 \%$ of simulations with a second wave of infection shown in Fig. 9a. These simulations result in an average of $\sim 300$ deaths on average. The majority of the simulations have only one wave of infection and result in an average of $\sim 150$ deaths, shown in Fig. 9b. This scenario results in $\sim 160$ deaths overall.

We also try the case where lockdown is from day 5 to day 65 and we resume to unrestricted interaction on day 110 (Fig. 10). In this case, the probability of a second wave of infection is $\sim 3 \%$-this case is shown in Fig. 10a. These simulations result in an average
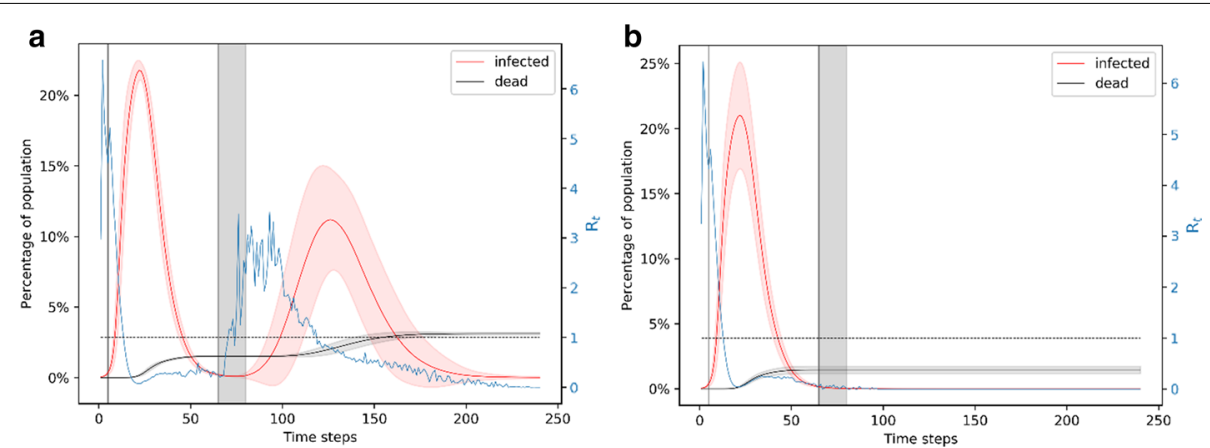

Fig. 8 Simulation of the case of a 15-day phasing out of the lockdown. A. Representation of the $19 \%$ of simulation results that have a second wave of infection if the lockdown is from day 5 to day 65 and complete normalcy resumes on day 80 . The effective reproduction number starts to increase during the phasing out of the mitigation measures. B. Representation of the $\sim 43 \%$ of simulation results that have only one wave of infection if the lockdown is from day 5 to day 65 and complete normalcy resumes on day 80. Comparing with Fig. 5, we note that a gradual phasing-out of the lockdown reduces the chances of a second wave of infection 

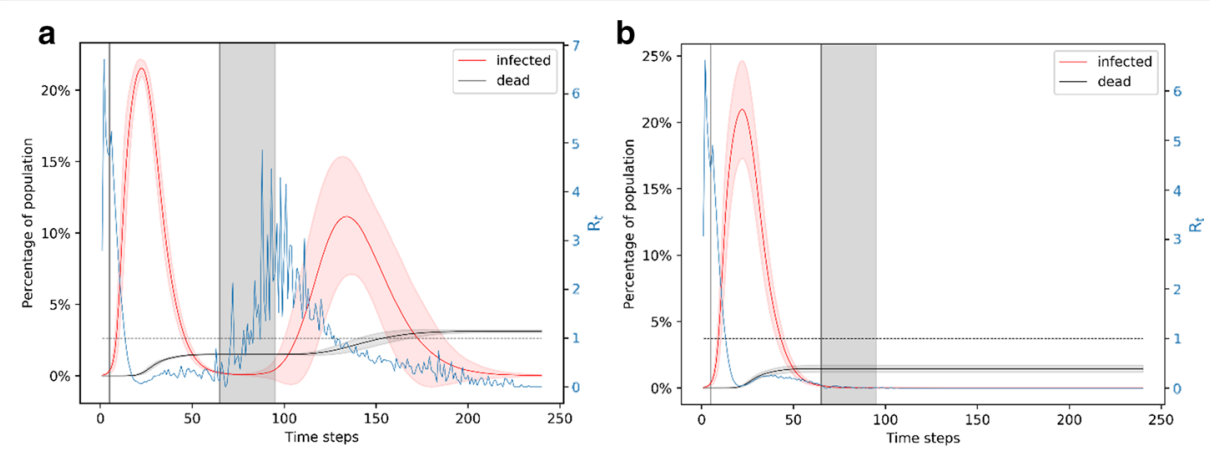

Fig. 9 Simulation of the case of phasing out of lockdown for 30 days. a Representation of the $6 \%$ of simulation results that have a second wave of infection for the lockdown from day 5 to day 65 and complete normalcy resumes on day 95 . b Representation of the $\sim 57 \%$ of simulation results that have only one wave of infection for the lockdown from day 5 to day 65 and complete normalcy resumes on day 95 . An increase in the duration of the phasing out period from 15 (see Fig. 8) to 30 leads to a significant reduction in the probability of a second wave of infection
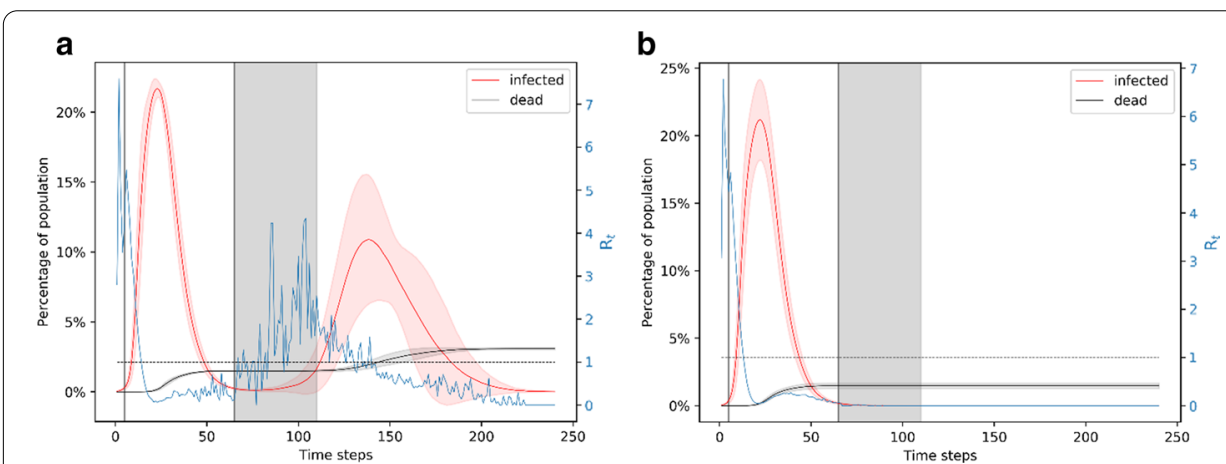

Fig. 10 Simulation of the case of phasing out of lockdown for 45 days. a Representation of the $~ 3 \%$ of simulation results that have a second wave of infection for the lockdown from day 5 to day 65 and complete normalcy resumes on day 110 . b Representation of the $59 \%$ of simulation results that have only one wave of infection for the lockdown from day 5 to day 65 and complete normalcy resumes on day 110. The 45-day phasing-out period after the lockdown is lifted makes a second wave of infection very unlikely

of $\sim 300$ deaths. The majority of the simulations, shown in Fig. 10b have only one wave of infection and result in an average of $\sim 150$ deaths. Overall, this scenario results in $\sim 150$ deaths. Similar to the case presented in the previous subsection, the effective reproduction number for all of these cases has at least one peak with value $\sim 6$ occurring around day 3-4 and has another peak for the results that show a second wave of infection. The $R_{t}$ peak is around 20 days before the peak of the second wave of infection. With the much lower probability of a second wave of infection and fewer deaths, we can conclude that a 60-day lockdown with an extended period of gradual decrease of mitigation would be the best approach.

\section{Discussion}

We showed that a simple network model results in insightful modeling of the spread of an infectious disease like Covid-19 under social distancing conditions. The network generation method is based on parsimonious assumptions on the nature of 
everyday interaction and combine different walks of human interaction. We combine existing network models such as random graphs and scale-free networks, to construct an image of the real-world interaction network. The variable parameters in our network generation method allow for modeling different kinds of communities. As more information about the transmission of Covid-19 becomes available, the network can be adjusted to focus on the interactions that are most conducive of transmission. For example, recent results highlight the importance of transmission in hospitals (Hâncean et al. 2020). Interactions with and among health care workers are included in our network's essential workplace interactions; these interactions could be considered separately to highlight their importance.

We also employ a simple disease spread mechanism in a network. Avoiding the complexity of more detailed spread processes that involve a latent period and presymptomatic phase, we employ few parameters to incorporate the most salient features of Covid-19 spread. As more precise information becomes available, the parameters can be tuned and the assumptions can be made more precise.

Our results show the trends of the probability of a second wave of infection and number of deaths for different lockdown situations. We explore different time-periods for lockdown starting with what is intuitively the safest option - a long lockdown window. We then explore shorter lockdown windows which give a moderately high probability for a second wave of infection. We explore different periods of gradual decrease of mitigation measures and find that a 60-day lockdown window paired with a 30-day or more of gradual increase of transmission leads to a very low probability of a second wave of infection and hence a lower death rate. Various modifications of this phasing out period could form promising directions for future work. For example, we can emulate the phasing out period by selectively adding edges to the sparser lockdown network instead of a varying transmission rate.

We also present the reproduction number of the disease calculated by case counting of the infection spread in the disease. There are various methods for estimating $\mathrm{R}_{\mathrm{t}}$ based on data-fitting and Monte Carlo simulations (Ma 2018; Inglesby 2020; Liu 2018; Aleta et al. 2020). For example, one can fit the number of infectious individuals to a Poisson distribution and then use Markov Chain Monte Carlo to obtain a distribution of $R_{t}$ (Aleta et al. 2020). The estimates on the basic reproduction number of Covid-19 (prior to mitigation) are in the range 4 to 8 ; the estimated effective reproduction number reduces to $\sim 1$ or less than 1 after 30 days of lockdown (Inglesby 2020; Aleta, et al. 2020; Rt COVID-19 2020; Gunzler and Sehgal 2020). In (Gunzler and Sehgal 2020), for example, they show that in the District of Columbia the value of $R_{t}$ on March 17 was 8.19 and on April 1 it was 1.00. Thus, our simulation results for the basic and effective reproduction numbers are consistent with the literature. Our results show that the peak in the reproduction number foreshadows the peak in the number of infected nodes (see Figs. 3, 4, 5, 6, 7, 8, 9, 10). Given the foreshadowing of the infection peak by the $\mathrm{R}_{\mathrm{t}}$ peak, an adaptive mitigation strategy may be useful, namely monitoring the rise in the effective reproduction number and accordingly implementing social distancing regulations for the population. 
Abbreviations

SARS-CoV-2: Severe acute respiratory syndrome coronavirus 2; Covid-19: Corona virus disease 2019.

Acknowledgements

We thank Jorge G.T. Zanudo, Jordan Rozum, Xiao Gan, and Dávid Deritei for helpful discussions.

\section{Authors' contributions}

PM and RA designed the research and methodology and PM performed the analyses. PM wrote the paper and both authors edited the paper. All authors read and approved the final manuscript.

\section{Funding}

This study was funded by NSF Grants MCB-1715826 and IIS-1814405 to RA.

\section{Availability of data and materials}

The code for network generation and analysis is available on Github repository: https://github.com/parulm/sprea d_lockdown.

\section{Competing interests}

The authors declare that they have no conflict of interest.

\section{Author details}

${ }^{1}$ Department of Physics, The Pennsylvania State University, University Park, PA 16802, USA. ${ }^{2}$ Biology Department, The Pennsylvania State University, University Park, PA 16802, USA

Received: 20 September 2020 Accepted: 8 December 2020

Published online: 29 December 2020

\section{References}

Aleta A et al (2020) Modeling the impact of social distancing, testing, contact tracing and household quarantine on second-wave scenarios of the COVID-19 epidemic. medRxiv

Barabási A-L, Albert R (1999) Emergence of scaling in random networks. Science 286(5439):509-512

Block P et al (2020) Social network-based distancing strategies to flatten the COVID-19 curve in a post-lockdown world. Nat Hum Behav 2020:1-9

Chen Y-C et al (2020) A time-dependent SIR model for COVID-19 with undetectable infected persons. Preprint arXiv :2003.00122

Chinazzi M et al (2020) The effect of travel restrictions on the spread of the 2019 novel coronavirus (COVID-19) outbreak. Science 368(6489):395-400

D'Souza G, Dowdy D (2020) What is herd immunity and how can we achieve it with COVID-19. https://www.jhsph.edu/ covid-19/articles/achieving-herd-immunity-with-covid19.html. Accessed 10 June 2020

Employment-to-Population Ratio (2020a) US Bureau of Labor Statistics. https://www.bls.gov/charts/employment-situa tion/employment-population-ratio.htm

Employment-to-Population Ratio (2020b). https://en.wikipedia.org/wiki/Employment-to-population_ratio. Accessed 10 June 2020

Erdős P, Rényi A (1960) On the evolution of random graphs. Publ Math Inst Hung Acad Sci 5(1):17-60

Gaubert S et al (2020) Forecasting the local progression of the Covid-19 epidemic from medical emergency calls: the example of the Paris area. Preprint arXiv:2005.14186

Gunzler D, Sehgal AR (2020) Time-varying COVID-19 reproduction number in the United States. medRxiv

Gutin G et al (2020) The effect of social distancing on the reach of an epidemic in social networks. Preprint arXiv :2005.03067

Hâncean M-G, Matjaž P, Lerner J (2020) Early spread of COVID-19 in Romania: imported cases from Italy and human-tohuman transmission networks. R Soc Open Sci 7(7):200780

Hopkins J (2020) CSSE coronavirus COVID-19 global cases (dashboard). https://coronavirus.jhu.edu/map.html. Accessed 6 June 2020

Inglesby TV (2020) Public health measures and the reproduction number of SARS-CoV-2. Jama

Kraemer MUG et al (2020) The effect of human mobility and control measures on the COVID-19 epidemic in China. Science 368(6490):493-497

Liu Q-H et al (2018) Measurability of the epidemic reproduction number in data-driven contact networks. Proc Natl Acad Sci 115(50):12680-12685

Ma Y et al (2018) Quantifying TB transmission: a systematic review of reproduction number and serial interval estimates for tuberculosis. Epidemiol Infection 146(12):1478-1494

O'neill BC, Chen BS (2020) Distribution of households in the United States from 1970 to 2019, by house size. https://www statista.com/statistics/242189/disitribution-of-households-in-the-us-by-household-size/. Accessed 10 June 2020

R. COVID-19 (2020) https://rt.live/. Accessed June 2020

Toda AA (2020) Susceptible-infected-recovered (sir) dynamics of covid-19 and economic impact. Preprint arXiv :2003.11221

\section{Publisher's Note}

Springer Nature remains neutral with regard to jurisdictional claims in published maps and institutional affiliations. 